\title{
The Effect of Helium Implantation on the Deformation Behaviour of Tungsten: X-ray Micro-diffraction and Nanoindentation
}

\author{
S. Das ${ }^{\mathrm{a}}$, D.E.J. Armstrong ${ }^{\mathrm{b}}$, Y. Zayachuk ${ }^{\mathrm{b}}$, W. Liu ${ }^{\mathrm{c}}$, R. Xuc ${ }^{\mathrm{c}}$, F. Hofmann ${ }^{\mathrm{a} *}$ \\ ${ }^{a}$ Department of Engineering Science, University of Oxford, Parks Road, Oxford OX1 3PJ, UK \\ ${ }^{b}$ Department of Materials, University of Oxford, Parks Road, Oxford OX1 3PH, UK \\ ${ }^{c}$ Advanced Photon Source, Argonne National Lab, 9700 South Cass Avenue, Argonne, IL 60439, \\ USA \\ *felix.hofmann@eng.ox.ac.uk
}

\begin{abstract}
:
The effect of helium-implantation-induced defects on deformation behaviour is examined by comparing spherical nano-indents in unimplanted and helium-implanted regions of a tungsten single crystal. Helium-implantation increases hardness and causes large pileups. 3D-resolved Xray micro-diffraction uniquely allows examination of the complex lattice distortions beneath specific indents. In the ion-implanted material we find reduced lattice rotations and residual strains due to indentation, indicating a more confined plastic zone. Together, our observations suggest that helium-induced defects initially act as efficient obstacles to dislocation motion, but are weakened by the subsequent passage of dislocations, causing a reduction in work hardening capacity.
\end{abstract}

\section{Main text:}

Tungsten is a promising candidate material for plasma facing components in future fusion reactors due to its high melting point, its strength at high temperatures and low sputtering yield [1-3]. However, challenges arise from its low fracture toughness, high brittle to ductile transition temperature (BDTT) [4] and poor machinability.

In the fusion reactor environment, armour components will be exposed to high temperatures ( $>$ $900{ }^{\circ} \mathrm{C}$ ) and bombardment with high-energy fusion neutrons (14.1 MeV) [3]. Interaction of these energetic neutrons with the tungsten lattice leads to collision cascades and the subsequent formation of defects in the material [5]. Furthermore, it causes transmutation, resulting in the formation of rhenium, osmium and tantalum, as well as hydrogen $(\mathrm{H})$ and helium $(\mathrm{He})$ [6-7]. At the anticipated operating temperatures, interstitial mobility of these gasses is high, allowing them to diffuse to defects, cracks or grain boundaries, resulting in embrittlement and hardening [8]. Hydrogen and helium will also be introduced into the material at plasma-facing surfaces exposed to large fluences of both elements [9-10]. 
The defect microstructure in tungsten is heavily influenced by the strong binding of helium to vacancies [11]. Previously nano-indentation showed a substantial increase in the hardness of tungsten after helium-ion implantation [12]. Interestingly helium ion implantation had a much greater effect on hardness than collision cascade damage caused by self-ion bombardment. This suggests that the presence of helium enhances defect retention and/or increases obstacle strength [12].

The effect of helium implantation on the mechanical properties of tungsten is potentially life limiting for the divertor, with significant economic consequences for fusion power. Resolving this problem requires a clear picture of the mechanisms governing the interaction of implantation defects with dislocation-mediated inelastic deformation. Experimentally these interactions are challenging to probe and at present remain poorly understood.

In this study, we consider spherical nano-indents in helium-implanted and unimplanted regions of the same tungsten single crystal, enabling a direct comparison and ensuring that any observed changes between the two regions are solely due to helium-implantation-induced defects. Using synchrotron X-ray micro-diffraction we are able to probe the lattice rotations and residual lattice strains beneath specific indents. These measurements uniquely provide access to the full, complex 3D lattice distortion field, whereas FIB lift-out and SEM/TEM observations, for example, only capture one slice though the indent. Importantly, since these X-ray measurements are non-destructive, the residual stress state remains unaltered. This 3D information is essential to unambiguously assess the underlying deformation fields that lead to the changes in surface morphology examinable by AFM or SEM (see exemplary finite element simulation in supplementary section). Our results reveal striking differences that are discussed in terms of the interaction between helium-induced defects and dislocations.

A $5 \mathrm{~mm}$ diameter, $0.8 \mathrm{~mm}$ thick disk with [001] out-of-plane orientation was cut from a tungsten single crystal (99.99\% purity). The [001] surface was mechanically ground and then polished using diamond paste. A final chemo-mechanical polishing step with $0.1 \mu \mathrm{m}$ colloidal silica suspension produced a high quality surface finish.

Part of the sample was implanted with helium ions at $298 \mathrm{~K}$ using a $2 \mathrm{MeV}$ ion accelerator at the National Ion Beam Centre, University of Surrey, UK. Implantation was carried out at room temperature $(298 \mathrm{~K})$ and using a raster scanned beam to ensure a uniform implantation dose. A combination of different ion energies and fluences was used to obtain a near uniform helium ion concentration in excess of 3000 appm within a $-2.8 \mu \mathrm{m}$ thick implanted layer [8,13 -14]. Fig. 1 (a) shows the implantation profile estimated using the SRIM code [15] (displacement energy of $68 \mathrm{eV}$, single-layer calculation model [16]). Between 0 and $2.8 \mu \mathrm{m}$ depth, a helium ion concentration of $\sim 3110 \pm 270 \mathrm{appm}$ is obtained with an associated damage of $0.24 \pm 0.02$ displacements per atom (dpa). Recoils induced by helium implantation have predominantly low energy, suggesting Frenkel pair formation as the main damage mechanism [14]. Since vacancy mobility is low at room temperature little defect clustering is expected [12,17-19]. 

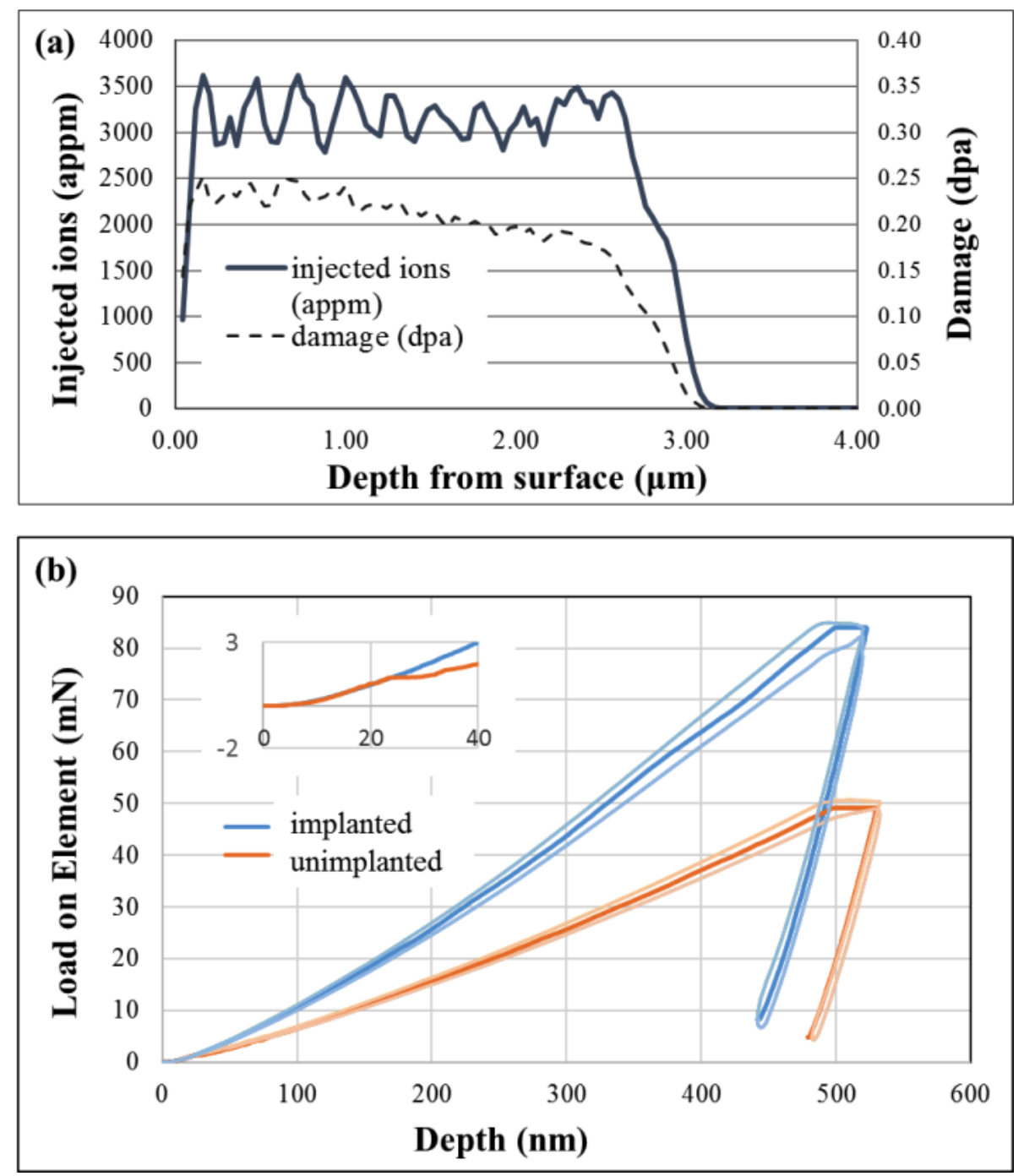

Figure 1 - (a) Profile of injected helium ion concentration calculated by SRIM (solid line) and the anticipated displacement damage (dashed line) plotted as a function of depth in the sample. (b) Load versus displacement curves for spherical indentation of the unimplanted and heliumimplanted sample (faint lines $=$ mean +- one standard deviation computed over 16 indents).

Nanoindentation was performed using an MTS NanoXp with a spherical, $\sim 4.2 \mu \mathrm{m}$ radius diamond indenter tip. Indents were made to a maximum displacement of $500 \mathrm{~nm}$ with a $50 \mu \mathrm{m}$ spacing between indents. Use of a spherical, axis-symmetric indenter removes the additional complexity, associated with in-plane indenter orientation relative to the crystal, found in Berkovich indentation. Fig. 1 (b) shows the load-displacement curves of the two indents considered in more detail below.

The load displacement curves show an initial Hertzian elastic response that is almost identical in the unimplanted and implanted material. This is as expected since ion-implantation-induced 
changes in elastic modulus are small $[14,20]$. At $\sim 25 \mathrm{~nm}$ depth the unimplanted sample shows a large pop-in, indicative of dislocation nucleation in the initially relatively defect free material at the onset of plastic deformation [21]. In the implanted sample, there are no obvious pop-ins. This indicates an easier nucleation of initial dislocation loops due to the presence of heliumimplantation-induced defects. At larger indentation depths the implanted sample shows a significantly greater indentation load than the unimplanted material, suggesting a substantial hardening due to helium-implantation-induced defects. This behaviour agrees well with previous Berkovich indentation of helium-implanted tungsten [12-13,22].

SEM micrographs of the nano-indents, recorded using a Zeiss Merlin FEG SEM, are shown in Fig. 2 (a) and (b). Little pile up is present in the unimplanted sample. The implanted material, on the other hand, shows a much larger, localised pile up around the indent tip. A similar trend was observed by Beck et al. who reported a substantial increase in pile up around Berkovich indents in helium-implanted tungsten of unidentified orientation [22]. Unfortunately, in their work the relative contributions of ion-implantation defects and indentation geometry to this effect could not be distinguished. Interestingly, in our study, the effects of crystallography are clearly visible: The pileup shows a strong dependence on the in-plane crystal orientation. Along $<100>$ directions the pileup is relatively small, whilst along $<110>$ directions a larger pileup is present. This is very clear in the implanted sample, where, due to the orientation dependent large pile up, the indenter impression assumes a square shape (sides of the square are along the $<100>$ direction). Such pile up patterns, localised in defined zones around the indent, and exhibiting a four-fold symmetry of out of plane displacement profile, have been previously observed in nanoindented (001) oriented $\mathrm{Cu}$ single crystals [23-24]. It can further be noted that the pileup in the implanted material shows a number of distinct steps indicated by small arrows in Fig. 2 (b). This suggests a slip localisation, similar to the dislocation channel formation reported in irradiationdamaged fcc metals [25-26].

Micro-beam Laue diffraction measurements were carried out at beamline 34-ID-E, Advanced Photon Source, Argonne National Lab, USA. This instrument allows measurements of lattice orientation with sub-micron 3D resolution using the Differential Aperture X-ray Microscopy (DAXM) technique. A detailed description of the DAXM technique and the 34-ID-E instrument is provided elsewhere [27-29]. Here a polychromatic X-ray beam $(5-30 \mathrm{keV})$, focussed to a size of $\sim 500 \mathrm{~nm}$, was used and measurements were carried out to a depth of $20 \mu \mathrm{m}$ beneath the ion-implanted surface. Laue diffraction patterns contained $30+$ peaks and were indexed and fitted using the LaueGo software package (J.Z. Tischler: tischler@anl.gov) to extract both lattice orientation and the full deviatoric lattice strain tensor at each measurement point in 3D space. 


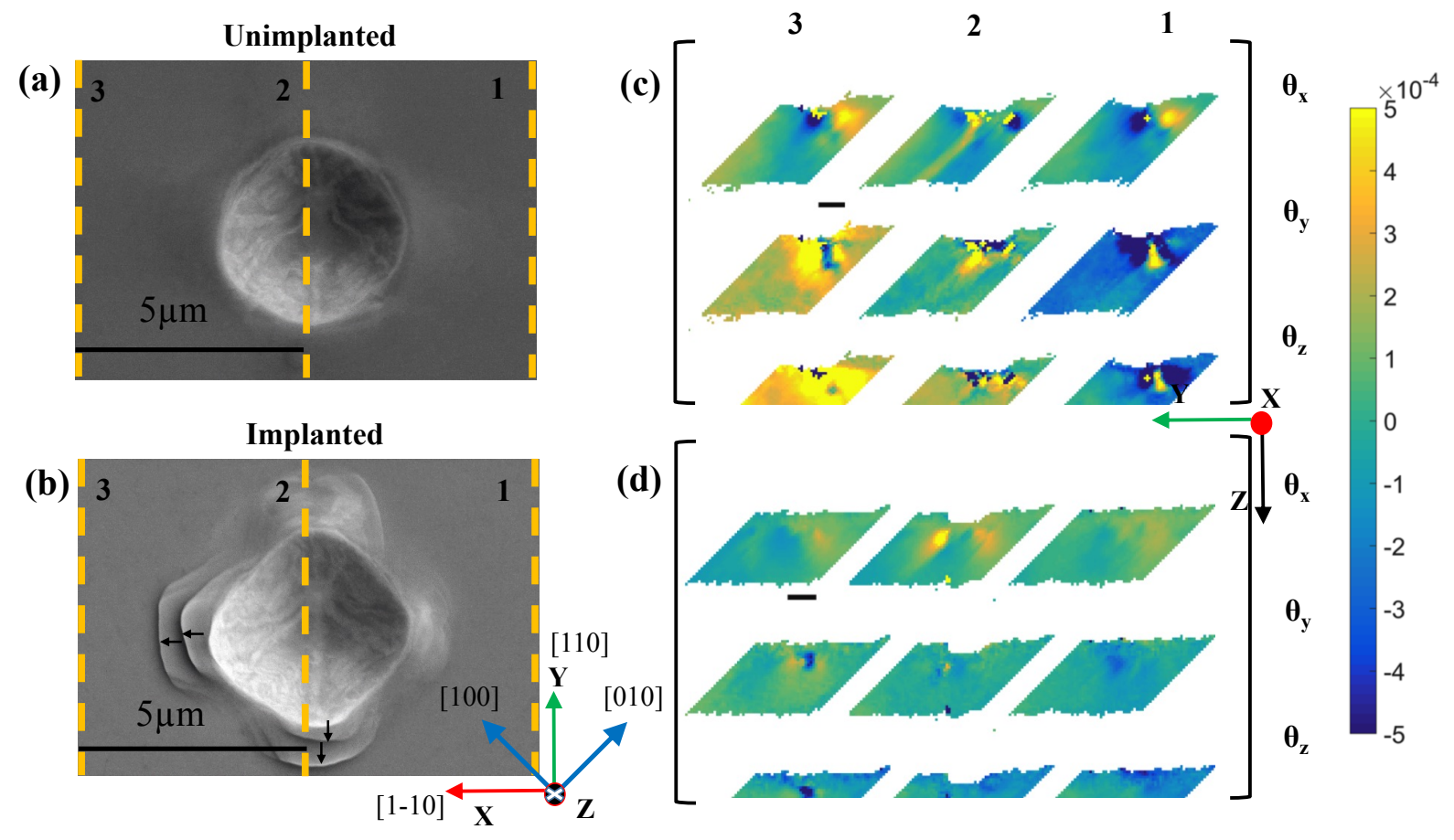

Figure 2 - (a) and (b) SEM micrographs of the sample with the $X, Y, Z$ coordinate frame superimposed and showing the relative orientations of [100] and [010] crystal axes. (a) shows an indent in the unimplanted region, whilst (b) shows an indent in the implanted material. (c) and (d) Lattice rotations in radians about the $X, Y$, and $Z$ axes (rows) shown on virtual $Y$ - $Z$ slices through the sample at 3 different $X$ positions for each indent. The locations of slices are shown as yellow lines in the SEM micrographs. Scale-bars correspond to $5 \mu \mathrm{m}$.

Lattice rotations, $\theta_{\mathrm{x}}, \theta_{\mathrm{y}}$ and $\theta_{\mathrm{z}}$, due to nano-indentation, refer to changes in crystal orientation compared to the pristine (unimplanted and undeformed) single crystal. The right-handed coordinate frame used here, and the corresponding crystal directions, are shown in Fig. 2 (a) and (b). Fig. 2 (c) and (d) show lattice rotation maps about all three axes for indents in the unimplanted and implanted material respectively. These rotations are plotted on virtual YZ slices through the sample at three X positions (indicated by dashed yellow lines Fig. 2 (a) and (b)). Positions 1 and 3 correspond to slices $5 \mu \mathrm{m}$ before and after the indent centre respectively, while position 2 corresponds to a slice through the indent centre.

In the unimplanted sample, lattice rotations are significantly larger than in the implanted material. However, both show similar patterns of lattice rotation variation at the different $\mathrm{X}$ positions (seen clearly in Fig. 4 in the supplementary section). As expected $\theta_{\mathrm{y}}$ is negative (anticlockwise rotation) before the indent centre (position 1) and positive after the indent centre (position 3). For $\theta_{\mathrm{x}}$ a similar pattern is observed with a positive lobe to the right and a negative lobe to the left at positions 1 and 3. At the indent centre, rather than clear positive and negative lobes, a rapidly varying arrangement of lattice rotations is observed beneath the indent. 
A common assumption in spherical indentation is that, compared to sharper indenters, there is a smooth, well-defined stress state under the indenter tip [30,31]. In fact, tungsten, being almost perfectly elastically isotropic, is perfect for illustrating this. However, the measured lattice rotations show that in fact even a small amount of plasticity can lead to much more complex lattice distortions. At the indent centre, the implanted and unimplanted materials show completely different lattice rotation structures. The magnitude of lattice rotations far from the indent centre is substantially larger in the unimplanted than the implanted material, suggesting a more confined plastic zone in the implanted case.

Strain information was extracted from the Laue diffraction data using the method described by Chung and Ice [32]. As the lattice strain tensor is symmetric, six strain components are shown in Fig. 3. Fig. 3 (a) and (b) show visualizations of the measured sample volume, coloured according to the diffracted intensity. The indent centre is indicated by the intersection of the $\mathrm{XZ}$ and $\mathrm{YZ}$ planes superimposed on Fig. 3 (a) and (b). Experimentally measured lattice strains on these planes in the unimplanted and implanted sample are shown in Fig. 3 (c) - (f). Strain plots for the XZ plane are shown in supplementary Fig. 5.

The differences in the residual elastic strain fields beneath the indent in the unimplanted and implanted material are striking. Strain magnitudes, particularly for the principal strain components and the $\varepsilon_{\mathrm{xy}}$ shear component, are significantly smaller in the implanted sample, suggesting a more confined plastic zone. The trend of compressive and tensile strains is similar in both, with the unimplanted sample showing large strain fields even far away from the indent centre. In the XY plane (parallel to the sample surface Fig. 3 (e) - (f)), four-fold symmetry of the strain distribution can be seen, consistent with the pileup patterns in SEM micrographs (Fig. 2 (a) and (b)). 
Unimplanted

(a)

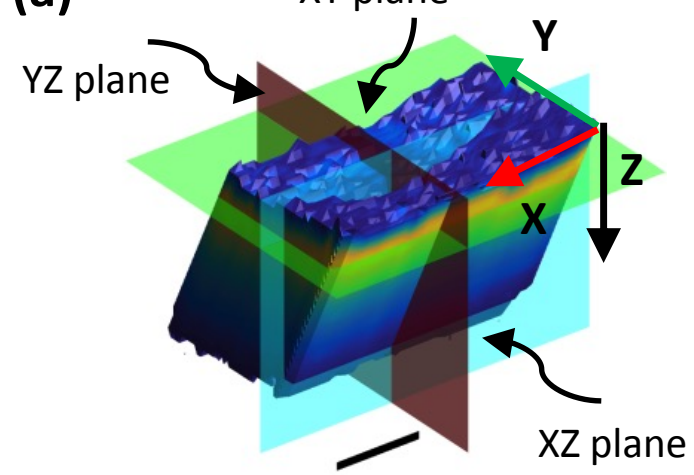

(c)

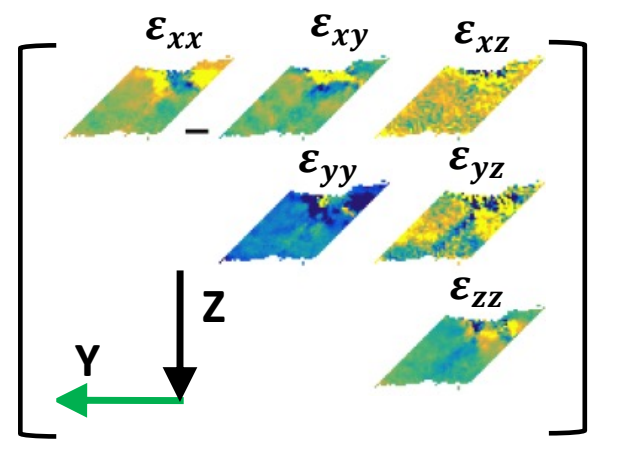

(b)

\section{Implanted}

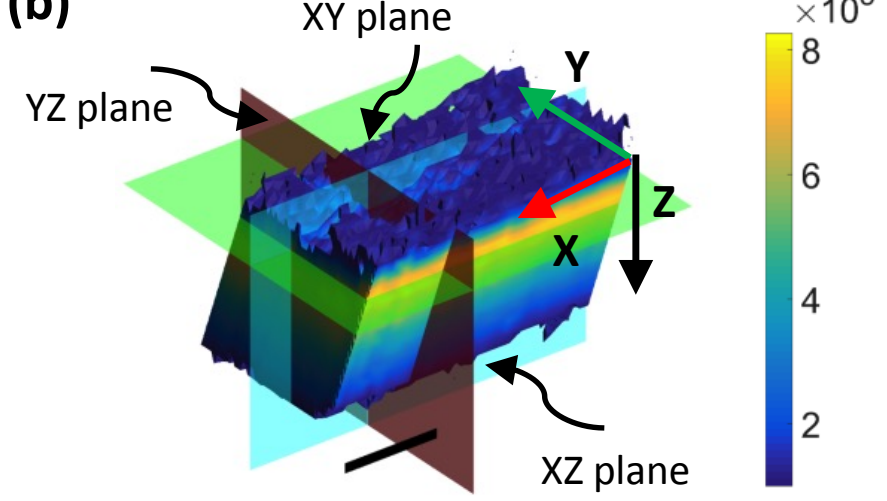

(d)

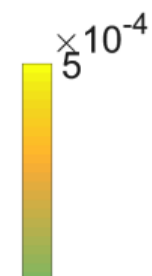

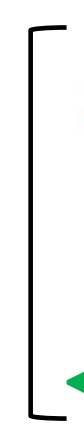

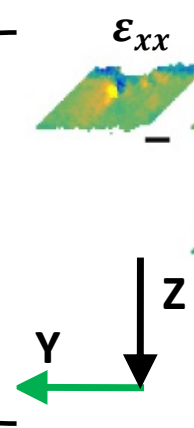

$\varepsilon_{x y}$

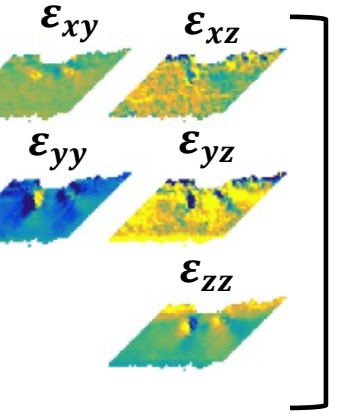

(f)
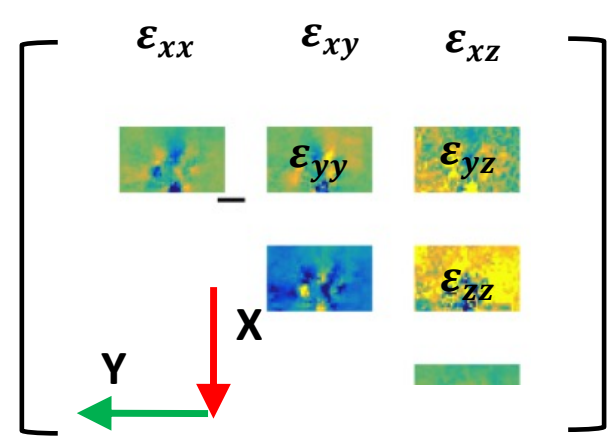

(e)

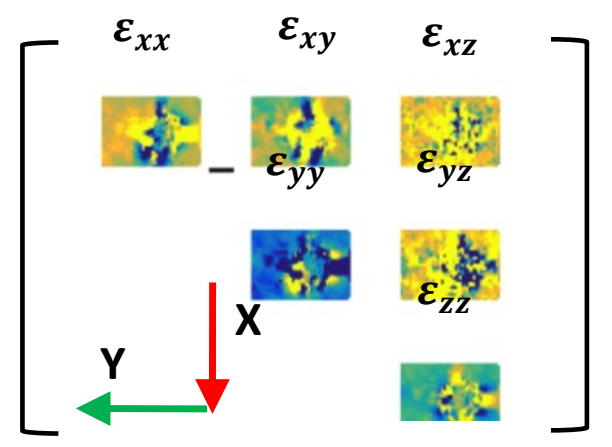

Figure 3 - (a) and (b) Three-dimensional rendering of the measured sample volume, coloured by scattered intensity. The top of the rendered volume corresponds to the sample surface, parallel to the $X Y$ plane. The intersection of $X Z$ and $Y Z$ planes marks the indent centre. Six components of the measured deviatoric elastic strains tensor plotted on the YZ plane, (c) and (d), and on the XZ plane, (e) and (f). (a), (c) and (e) are for the unimplanted sample; (b), (d) and (f) are for the implanted sample. Scale-bars correspond to $5 \mu \mathrm{m}$.

Implantation with helium results in the formation of small helium-vacancy clusters to which the self-interstitial atoms remain strongly attached [14,33]. The greater hardness of the implanted material shows that small He-vacancy clusters, unresolvable by TEM [12], act as surprisingly strong obstacles to dislocation motion. Here the presence of slip steps in the implanted material is interesting, as it indicates a localization of deformation and supports the formation of 
dislocation channels. We hypothesize that gliding dislocations help to recombine vacancies and self-interstitial atoms, thus sweeping out channels with reduced defect density in which subsequent dislocations can propagate more easily, thus resulting in slip-step formation.

In other neutron-irradiated bcc materials, for instance vanadium, dislocation channels have been observed on $\{112\}$ and $\{110\}$ planes, even at low damage levels of 0.012 dpa [25]. The slip steps observed here in $\langle 110\rangle$ directions in Fig. 2 (b) are consistent with this. The formation of dislocation channels with reduced obstacle concentration has been previously reported to lead to reduced strain hardening capacity [34] or work softening [35]. This is consistent with the increase in pileup we observe in the implanted material. Indeed simulations by Oliver and Phar have previously linked increase pileup to reduced work hardening capacity [36].

In summary, we have observed localization of pileup, formation of dislocations channels and a more confined plastic zone associated with spherical nano-indents in helium-implanted tungsten. Our results suggest that helium-implantation-induced defects initially act as efficient obstacles to dislocation motion, but are removed by interaction with passing dislocations. This behaviour differs markedly from observations in heavy ion irradiated tungsten where the opposite effect, i.e. a suppression of pileup, has been reported [37]. This raises the question why the interaction of dislocations with helium filled implantation-induced defects is so different to interaction with defects without helium. The exact mechanism that governs this interaction and the fate of the helium remain unclear. We hope that these new detailed observations will stimulate the interest of the modelling community to elucidate the interaction of implantation-induced defects with glide dislocations. This is of particular importance since increased hardness and reduced work hardening capacity are detrimental to material ductility, a key concern for long-term structural integrity of future tungsten based diverter concepts.

\section{Acknowledgements:}

We thank Dr. E. Tarleton for insightful discussions, Dr. L. Hansen for providing the spherical indenter tip, Prof. M. Rieth for providing single crystal tungsten samples and Dr. N. Peng for performing the ion-implantation. This work was funded by Leverhulme Trust Research Project Grant RPG-2016-190. This research used resources of the Advanced Photon Source, a U.S. Department of Energy (DOE) Office of Science User Facility operated for the DOE Office of Science by Argonne National Laboratory under Contract No. DE-AC02-06CH11357. DEJA acknowledges the Royal Academy of Engineering for his Research Fellowship. This work has been carried out within the framework of the EUROfusion Consortium and has received funding from the Euratom research and training programme 2014-2018 under grant agreement No 633053. The views and opinions expressed herein do not necessarily reflect those of the European Commission. We acknowledge part funding from the UK Engineering and Physical Sciences Research Council, grant EP/H018921/1. 


\section{References}

[1] M. Ekman, K. Persson, G. Grimvall, J. Nucl. Mater. 278 (2000) 276-279.

[2] N. Wei, T. Jia, X. Zhang, T. Liu, Z. Zeng, X. Yang, AIP Adv 4. 057103 (2014).

[3] M. Rieth, S.L. Dudarev, S.M. Gonzalez de Vicente, J. Aktaa, T. Ahlgren, S. Antusch, D.E.J. Armstrong, M. Balden, N. Baluc, M.-F. Barthe, W.W. Basuki, M. Battabyal, C.S. Becquart, D. Blagoeva, H. Boldyryeva, J. Brinkmann, M. Celino, L. Ciupinski, J.B. Correia, A. De Backer, C. Domain, E. Gaganidze, C. García-Rosales, J. Gibson, M.R. Gilbert, S. Giusepponi, B. Gludovatz, H. Greuner, K. Heinola, T. Höschen, A. Hoffmann, N. Holstein, F. Koch, W. Krauss, H. Li, S. Lindig, J. Linke, C. Linsmeier, P. López-Ruiz, H. Maier, J. Matejicek, T.P. Mishra, M. Muhammed, A. Muñoz, M. Muzyk, K. Nordlund, D. Nguyen-Manh, J. Opschoor, N. Ordás, T. Palacios, G. Pintsuk, R. Pippan, J. Reiser, J. Riesch, S.G. Roberts, L. Romaner, M. Rosiński, M. Sanchez, W. Schulmeyer, H. Traxler, A. Ureña, J.G. van der Laan, L. Veleva, S. Wahlberg, M. Walter, T. Weber, T. Weitkamp, S. Wurster, M.A. Yar, J.H. You, A. Zivelonghi, J. Nucl. Mater. 432 (2013) 482-500.

[4] P. Gumbsch, J. Riedle, A. Hartmaier, H.F. Fischmeister, Science (80-. ). 282 (1998) $1293-1295$.

[5] X. Yi, M.L. Jenkins, M. Briceno, S.G. Roberts, Z. Zhou, M.A. Kirk, Philos. Mag. 93 (2013) 1715-1738.

[6] M.R. Gilbert, J.-C. Sublet, Nucl. Fusion 51 (2011) 43005.

[7] M.R. Gilbert, S.L. Dudarev, S. Zheng, L.W. Packer, J.-C. Sublet, Nucl. Fusion 52 (2012) 83019 .

[8] I. De Broglie, C.E. Beck, W. Liu, F. Hofmann, Scr. Mater. 107 (2015).

[9] Y. Zayachuk, M.H.J. 't Hoen, P.A. Zeijlmans Van Emmichoven, D. Terentyev, I. Uytdenhouwen, G. Van Oost, Nucl. Fusion 53 (2013) 13013-7.

[10] S. Sharafat, A. Takahashi, Q. Hu, N.M. Ghoniem, J. Nucl. Mater. 386-388 (2009) 900903.

[11] P.E. Lhuillier, T. Belhabib, P. Desgardin, B. Courtois, T. Sauvage, M.F. Barthe, A.L. Thomann, J. Nucl. Mater. 416 (2011) 13-17.

[12] D.E.J. Armstrong, P.D. Edmondson, S.G. Roberts, Appl. Phys. Lett. 251901 (2013) 1-5.

[13] C.E. Beck, S.G. Roberts, P.D. Edmondson, D.E.J. Armstrong, MRS Proc. 1514 (2013) 99-104.

[14] F. Hofmann, D. Nguyen-Manh, M.R. Gilbert, C.E. Beck, J.K. Eliason, A.A. Maznev, W. Liu, D.E.J. Armstrong, K.A. Nelson, S.L. Dudarev, Acta Mater. 89 (2015) 352-363.

[15] J.F. Ziegler, J. Biersack, Nucl. Inst. Methods Phys. Res. B 268 (2010) 1818-1823. 
[16] ASTM E521-96(2009) e1, Standard Practice for Neutron Radiation Damage Simulation by Charged-Particle Irradiation, ASTM International, West Conshohocken, PA, 2009, USA.

[17] A. Debelle, M.F. Barthe, T. Sauvage, J. Nucl. Mater. 376 (2008) 216-221.

[18] P.M. Derlet, D. Nguyen-Manh, S.L. Dudarev, Phys. Rev. B - Condens. Matter Mater. Phys. 76 (2007).

[19] D. Nguyen-Manh, A.P. Horsfield, S.L. Dudarev, Phys. Rev. B - Condens. Matter Mater. Phys. 73 (2006).

[20] R.A. Duncan, F. Hofmann, A. Vega-Flick, J.K. Eliason, A.A. Maznev, A.G. Every, K.A. Nelson, Appl. Phys. Lett. 109 (2016).

[21] L. Ma, L. Levine, R. Dixson, D. Smith, D. Bahr, Nanoindentation Mater. Sci. 22 (2012) 1656-1661.

[22] C.E. Beck, F. Hofmann, J.K. Eliason, A.A. Maznev, K.A. Nelson, D.E.J. Armstrong, D.E.J. Armstrong, Scr. Mater. 128 (2017) 83-86.

[23] D.G. Flom, R. Komanduri, Wear 252 (2002) 401-429.

[24] Y. Wang, D. Raabe, C. Klüber, F. Roters, Acta Mater. 52 (2004) 2229-2238.

[25] N. Hashimoto, T.S. Byun, K. Farrell, S.J. Zinkle, J. Nucl. Mater. 329-333 (2004) 947952.

[26] D. Kiener, P. Hosemann, S.A. Maloy, A.M. Minor, Nat. Mater. 10 (2011) 608-613.

[27] W. Liu, G.E. Ice, B.C. Larson, W. Yang, J.Z. Tischler, J.D. Budai, Metall. Mater. Trans. A 35 (2004) 1963-1967.

[28] W. Liu, P. Zschack, J. Tischler, G. Ice, B. Larson, in:, AIP Conf. Proc., 2010, 108-111.

[29] F. Hofmann, B. Abbey, W. Liu, R. Xu, B.F. Usher, E. Balaur, Y. Liu, Nat. Commun. 4 (2013) 2774.

[30] S. Pathak, S.R. Kalidindi, Mater. Sci. Eng. R 91 (2015) 1-36.

[31] S.R. Kalidindi, S. Pathak, Acta Mater. 56 (2008) 3523-3532.

[32] K.S. Chung, G.E. Ice, J. Appl. Phys. 86 (1999) 5249-5255.

[33] J. Boisse, C. Domain, C.S. Becquart, J. Nucl. Mater. 455 (2014) 10-15.

[34] P.M. Rice, S.J. Zinkle, J. Nucl. Mater. 258 (1998) 1414-1419.

[35] M.J. Makin, J. V. Sharp, Phys. Status Solidi 9 (1965) 109-118.

[36] W.C. Oliver, G.M. Pharr, J. Mater. Res. 19 (2004) 3-20.

[37] D.E.J. Armstrong, A.J. Wilkinson, S.G. Roberts, Phys. Scr. T145 (2011) 14076. 


\section{Supplementary Section}

\section{Calculated Lattice Orientation Changes}

Fig. 4 shows the changes in lattice rotations, plotted on the YZ plane, for all three components, $\theta_{\mathrm{x}}, \theta_{\mathrm{y}}$ and $\theta_{\mathrm{z}}$, in incremental positions along the $\mathrm{X}$ direction. Fig. 2 in the main text illustrates these changing lattice rotations on only three chosen slices, before and after the indent centre. Fig. 4 further elucidates the progressive evolution of the lattice rotations along the $\mathrm{X}$ direction by depicting it on $0.9 \mu \mathrm{m}$ incremental positions at distances of up to $5.4 \mu \mathrm{m}$ from the indent centre. Lattice rotations for the helium-implanted tungsten are displayed using a reduced colour-scale as compared to the unimplanted sample. This has been done to clearly show the pattern of the smaller lattice rotations caused by helium-implantation and to get a pronounced view of their progressive evolution along the $\mathrm{X}$-axis.

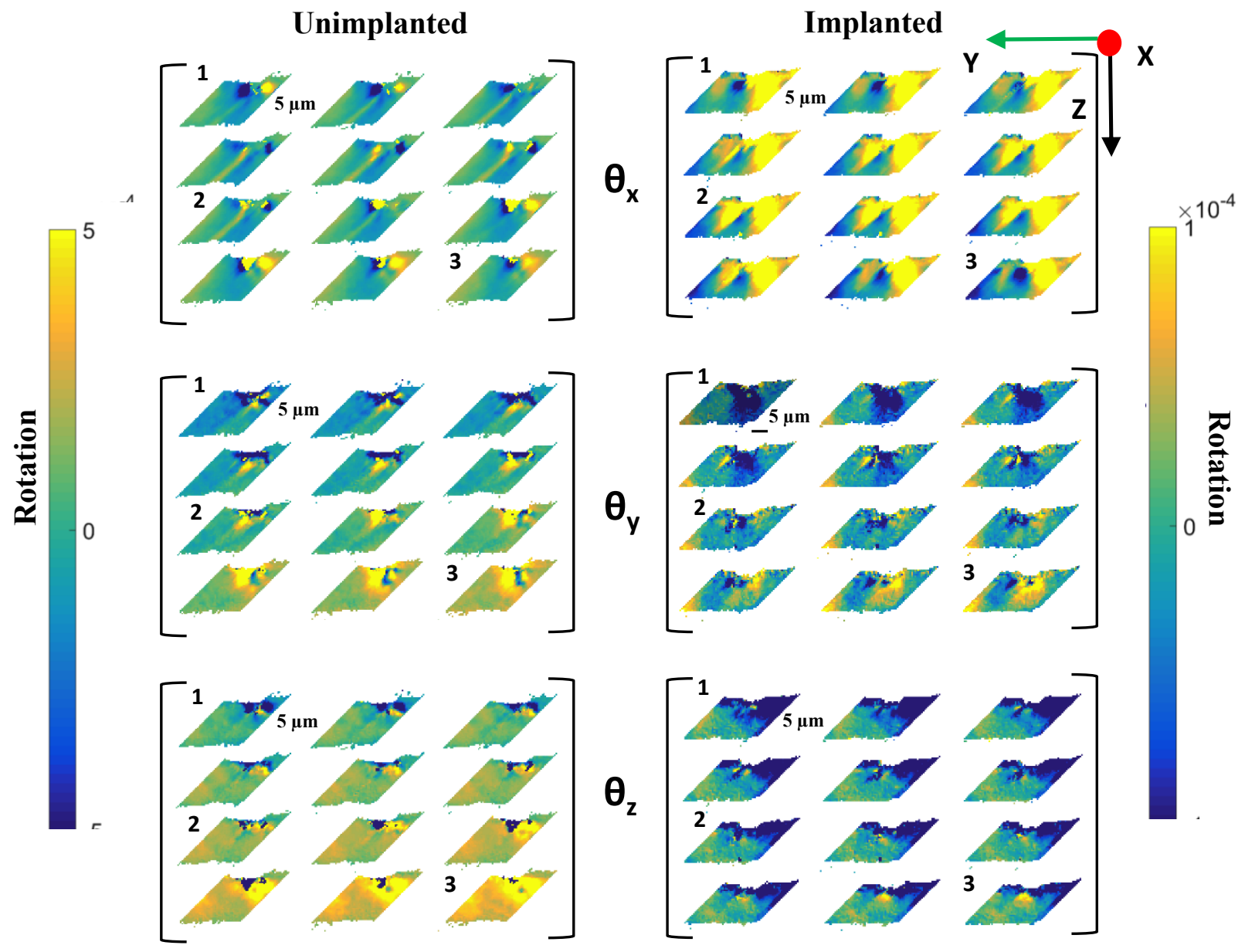

Figure 4 - Lattice rotations in radians at incremental positions (0.9 $\mu \mathrm{m}$ between slices) along the $X$ direction plotted on the YZ sample plane; Positions 1, 2 and 3 correspond to cross section slices shown in Fig. 2. Position 1 corresponds to a point on the X-axis before the indent centre, position 2 is at the indent centre and position 3 is a slice after the indent centre. Scale bars correspond to $5 \mu \mathrm{m}$. 


\section{Experimentally measured strains in the $\mathrm{XZ}$ plane}

Figure 5 shows the six components of the measured deviatoric lattice strain tensor, plotted on the $\mathrm{XZ}$ plane, at the indent centre. As observed in the YZ and XY planes (Fig. 3 in main text), the strain fields are larger in magnitude in the unimplanted sample than in the helium-implanted sample. This is particularly noticeable for the $\varepsilon_{\mathrm{xx}}, \varepsilon_{\mathrm{xy}}$ and the $\varepsilon_{\mathrm{zz}}$ components.

(a)

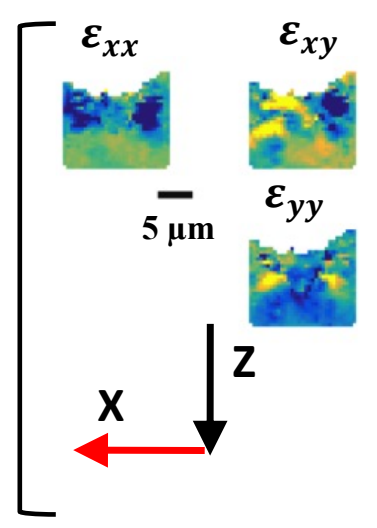

Unimplanted

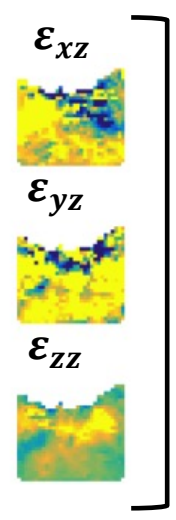

(b)

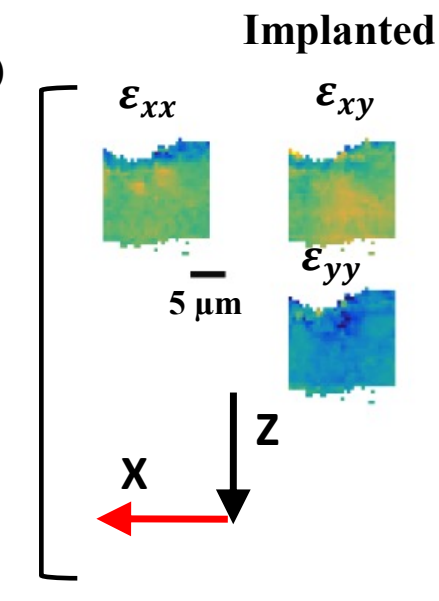

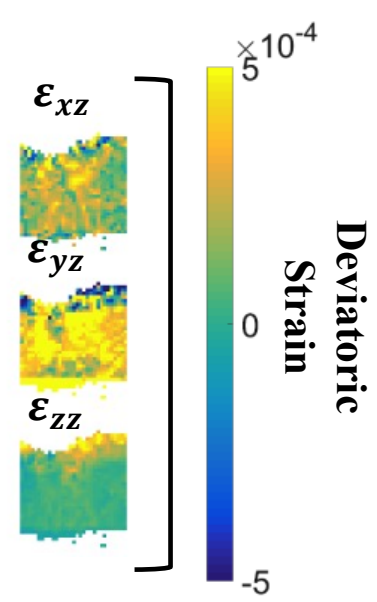

Figure 5 - Experimentally measured elastic strains at the indent centre on the XZ plane. Scale bars correspond to $5 \mu \mathrm{m}$.

\section{The need for 3D volume information to understand indentation-induced plasticity}

Although pile up and channel formation could be observed by SEM and AFM measurements, the spread of the plastic zone underneath the indent cannot be unambiguously concluded from these methods. A study by Kiener et al. [1], reviews the different characterization techniques (etch-pit, EBSD and in-situ TEM) used to analyse nano-indentation induced plasticity. Their report illustrates the limitations of each method (restricted to surface analysis, extensive sample preparation, lack of sensitivity to dislocations parallel to surface, restricted sample size etc.) and stresses on the need to use multiple techniques to fully understand the deformation mechanisms [1].

To illustrate why surface observations are not sufficient, the results from a simple 2D finite element simulation of a nano-indentation experiment are provided below. They show that different combinations of yield stress and hardening (Fig. 6 (a)) can result in very similar surface profiles (Fig. 6 (b)) but very different plastic deformation underneath the indent (Fig. 6 (c), (d)). 3D Laue micro diffraction measurements uniquely allow access to the complex 3D lattice distortion fields beneath specific indents without altering the residual stress state. 
(a)

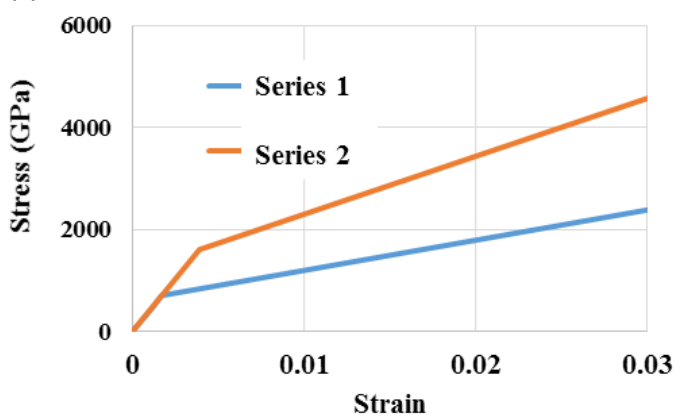

(c)

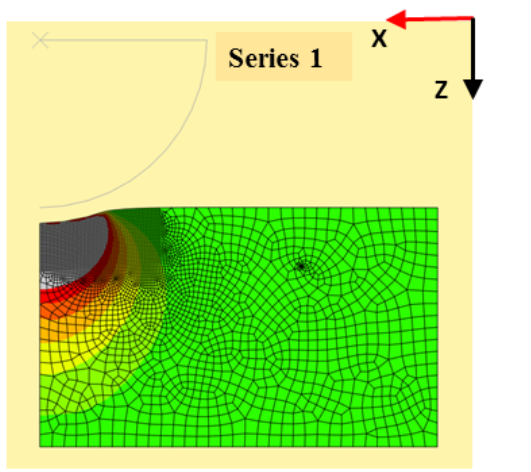

(b)

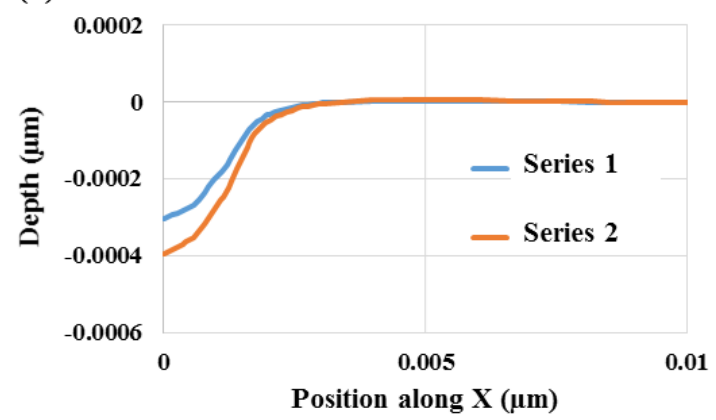

(d)

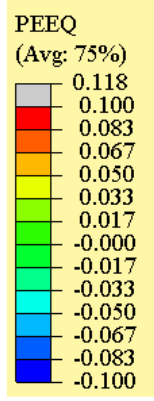

Series 2
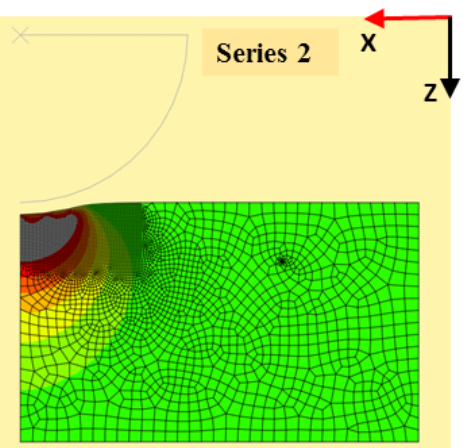

Figure 6 - (a) Stress-strain curves for 2 chosen values of yield stress and linear hardening; (b) Indent-depth profile for the 2 chosen sets of yield stress and linear hardening; The equivalent plastic strain field beneath the indent after unloading as simulated in Abaqus using a 2D axisymmetric model with Von Mises yield criterion and linear hardening (c) for Series 1 and (d) for Series 2.

\section{Supplementary References}

[1] D. Kiener, K. Durst, M. Rester, and A. M. Minor, "Revealing deformation mechanisms with nanoindentation," JOM, vol. 61, no. 3. pp. 14-23, 2009. 